\title{
Erratum to: Incidental Findings of Intense Radioiodine Uptake in Struma Ovarii and Bilateral Non-Lactating Breast Simultaneously on Post-Ablation SPECT/CT for Papillary Thyroid Cancer
}

\author{
Hye-kyung Shim ${ }^{1}$ - Mi Ra Kim ${ }^{2}$
}

Published online: 7 March 2017

(C) Korean Society of Nuclear Medicine 2017

\section{Erratum to: Nucl Med Mol Imaging}

DOI 10.1007/s13139-016-0459-0

\begin{abstract}
Although the corresponding author of article "Incidental findings of intense radioiodine uptake in struma ovarii and bilateral non-lactating breast simultaneously on post-ablation SPECT/CT for papillary thyroid cancer" is Mi Ra Kim, the name and e-mail address of the first author, Hye-kyung Shim, were incorrectly given instead (Nucl Med Mol Imaging (2016) 50: p353).

The publisher apologizes for this error and the inconvenience caused.
\end{abstract}

The online version of the original article can be found at http://dx.doi.org/ 10.1007/s13139-016-0459-0.

Mi Ra Kim

Enthns@hanmail.net

1 Department of Nuclear Medicine, Haeundae Paik Hospital, University of Inje, College of Medicine, 875 Haeun-daero, Haeundae-gu, Busan, South Korea

2 Department of Otorhinolaryngology-Head and Neck Surgery, Haeundae Paik Hospital, Inje University College of Medicine, Busan, South Korea 